\title{
FRACTIONAL PROGRAMMING DUALITY VIA GEOMETRIC PROGRAMMING DUALITY
}

\author{
C. H. SCOTT and T. R. JEFFERSON
}

(Received 13 September 1978)

(Revised 15 March 1979)

\begin{abstract}
A duality theory for a class of fractional programs is developed. A fractional program which is non-convex is convexified using a one-to-one transformation. The resulting convex equivalent is then dualized with generalized geometric programming duality.
\end{abstract}

\section{Introduction}

We consider the following fractional program:

$$
\begin{array}{ll}
\text { minimize } & g(x)=c(x) / f(x) \text { over } x \in C \subset R^{n} \\
\text { subject to } & A x \geqslant b
\end{array}
$$

where $c(\cdot)$ is a convex function defined on a closed convex set $C$ and $f(\cdot)$ is a linear function of the form $f_{1}^{\mathrm{T}} x+f_{0}$ which is assumed to be positive over the constraint set $\{x \in C \mid A x \geqslant b\} . A$ is a given $m \times n$ matrix, $b \in R^{m}, f_{1} \in R^{n}$ and $f_{0} \in R$ are given.

Considerable effort has been directed at constructing duals to non-convex mathematical programs of the fractional type $[1,5,9,12,3,7]$. However, most of this research has used Lagrangian ideas. Here, we propose to use the unconstrained version of generalized geometric programming [8] to construct a new dual to the fractional program posed above. This approach is valuable since it separates the primal and dual variables. The resulting convex program can then be approached independently of the primal nonconvex program.

A detailed discussion of the applications, theory and computational aspects of fractional programming has recently been given by Craven [4]. 


\section{Geometric duality}

The generalized theory of geometric programming [8] pairs the following two convex mathematical programs termed primal and dual respectively:

1.

Minimize $g(x)$ over $x \in C$

subject to $x \in \chi$.

2.

minimize $g^{*}\left(x^{*}\right)$ over $x^{*} \in C^{*}$

subject to $x^{*} \in \chi^{*}$.

Here $g(\cdot)$ is a convex function defined on a closed convex set $C \subset R^{n}, \chi$ is a closed convex cone in $R^{n}$, and $g^{*}(\cdot)$ defined on $C^{*}$ denotes the conjugate transform of $g(\cdot)$, that is,

and

$$
g^{*}\left(x^{*}\right)=\sup _{x \in C}\left(x^{\mathrm{T}} x^{*}-g(x)\right)
$$

$$
C^{*}=\left\{x^{*} \in R^{n} \mid \sup _{x \in C}\left(x^{\mathrm{T}} x^{*}-g(x)\right)<\infty\right\} .
$$

Also, $\chi^{*}$ is the dual cone of $\chi$, that is,

$$
\chi^{*}=\left\{x^{*} \mid x^{\top} x^{*} \geqslant 0 \text { for all } x \in \chi\right\} .
$$

At optimality, the following relations hold [8].

(i) $\min _{x \in \mathcal{C} n x} g(x)+\min _{x^{*} \in C^{*} n x^{*}} g^{*}\left(x^{*}\right)=0$,

(ii) $x \in \partial g^{*}\left(x^{*}\right), \quad x^{*} \in \partial g(x)$,

(iii) $x^{\mathrm{T}} x^{*}=0$.

Here $\partial g(x)$ denotes the subgradient set of $g(\cdot)$ at $x$, that is,

$$
\partial g(x)=\left\{x^{*} \mid\left\langle x^{*}, y-x\right\rangle \leqslant g(y)-g(x), \text { for all } y \in C\right\} .
$$

For the purposes of Section 3, we require the following definition of the positive homogeneous extension $c^{+}(x, \lambda), \lambda \in R$ of a convex function $c(x)$ defined on a closed convex set $C$ :

$$
c^{+}(x, \lambda)= \begin{cases}\sup _{x^{*} \in C^{*}} x^{\mathrm{T}} x^{*} & \text { if } \lambda=0 \text { and } \sup _{x^{*} \in C^{*}} x^{\mathrm{T}} x^{*}<\infty, \\ \lambda c(x / \lambda) & \text { if } \lambda>0 \text { and } x / \lambda \in C .\end{cases}
$$

This is defined on $C^{+}$where

$$
C^{+}=\left\{(x, \lambda) \mid \lambda=0 \text { and } \sup _{x^{*} \in C^{*}} x^{\mathrm{T}} x^{*}<\infty\right\} \cup\{(x, \lambda) \mid \lambda>0 \text { and } x / \lambda \in C\} \text {. }
$$

It has been shown that the conjugate transform of $c^{+}(x, \lambda)$ defined on $C^{+}$is the identically zero function defined on the convex set $c^{*}\left(x^{*}\right)+\lambda^{*} \leqslant 0[8]$. 


\section{Fractional duality}

A useful means of analysing a fractional program is to transform it into an equivalent convex program. This approach was initiated by Charnes and Cooper [2] for the case where $c(x)$ is linear. For general convex $c(x)$, Manas [6] has introduced the following one-to-one transformation of the form

$$
z_{0}=1 / f(x), \quad z=z_{0} x
$$

In the transform variables, the program defined by (1) and (2) is

$$
\text { minimize } \begin{aligned}
g\left(z, z_{0}\right) & =c\left(z / z_{0}\right) z_{0} \\
\text { subject to } \quad A z-b z_{0} & \geqslant 0, \\
f_{1} z+f_{0} z_{0} & =1, \\
z_{0} & >0,
\end{aligned}
$$

which is convex and equivalent to the original non-convex program [11].

We may close the functions without loss of generality in order to invoke the duality theory. Hence the above program may be written

where

$$
\begin{array}{ll}
\text { minimize } & c^{+}\left(z, z_{0}\right) \text { over }\left(z, z_{0}\right)^{\mathrm{T}} \in C^{+}, \quad \alpha \in\{1\}, \\
\text { subject to } & \left(z, z_{0}, \alpha\right) \in \chi
\end{array}
$$

$$
\chi=\left\{\left(z, \alpha, z_{0}\right) \mid A z-b z_{0} \geqslant 0, f_{1}^{\mathbf{T}} z+f_{0} z_{0}-\alpha=0\right\} .
$$

Here $\alpha$ is associated with an identically zero component of the objective function. We require the dual of the cone $\chi$, that is,

$$
\left\{\left(z^{*}, \alpha^{*}, z_{0}^{*}\right) \mid\left\langle z, z^{*}\right\rangle+z_{0} z_{0}^{*}+\alpha \alpha^{*} \geqslant 0 \text {, for all }\left(z, \alpha, z_{0}\right) \in \chi\right\} \text {. }
$$

This is readily found as

$$
\left\{\begin{array}{c}
z^{*} \\
\alpha^{*} \\
z_{0}^{*}
\end{array}\right\}=\left\{\begin{array}{c}
A^{\mathrm{T}} \\
0 \\
-b^{\mathrm{T}}
\end{array}\right\} u+\left(\begin{array}{c}
f_{1} \\
-1 \\
f_{0}
\end{array}\right\} v,
$$

where $u \in R^{m}$ and $u \geqslant 0, v \in R$.

Hence, following the prescription in Section 2, the dual program is

$$
\begin{array}{ll}
\text { minimize } & \alpha^{*} \\
\text { subject to } & c^{*}\left(z^{*}\right)-b^{\mathrm{T}} u-f_{0} \alpha^{*} \leqslant 0, \\
& z^{*}=A^{\mathbf{T}} u-f_{1} \alpha^{*}, \\
& u \geqslant 0 .
\end{array}
$$

Here $c^{*}\left(z^{*}\right)$ denotes the conjugate transform of $c(z)$. For the particular case that $c(z)$ is linear in $z$, these results are equivalent to those obtained by Charnes and Cooper [2]. However, for general convex $c(z)$, they are different from previous results $[1,3,5,7,9,12,13]$ since this duality separates primal and dual variables. 


\section{Acknowledgements}

The authors wish to acknowledge the helpful comments of the referees which have improved the presentation of the paper.

\section{References}

[1] C. R. Bector, "Duality in nonlinear fractional programming", Zeitschrift für Operations Research 17 (1973), 183-193.

[2] A. Charnes and W. W. Cooper, "Programming with linear fractional functionals", Nav. Res. Log. Quart. 9 (1962), 181-186.

[3] B. D. Craven and B. Mond, "The dual of a fractional linear program", J. Math. Anal. Appl. 42 (1973), 507-512.

[4] B. D. Craven, Mathematical programming and control theory (Chapman and Hall, 1978).

[5] R. Jagannathan, "Duality for nonlinear fractional programs", Zeitschrift für Operations Research 17 (1973), 1-3.

[6] M. Manas, "On transformation of quasi-convex programming problems", Ekonomicko Matematicky Obzor 4 (1968), 93-99.

[7] B. Mond and B. D. Craven, "A note on mathematical programming with fractional objective functions", Nav. Res. Log. Quart. 20 (1973), 577-581.

[8] E. L. Peterson, "Geometric programming", SIAM Review 18 (1976), 1-52.

[9] O. Rani and R. N. Kaul, "Duality theorems for a class of non-convex programming problems", J. Optim. Theory Applic. 11 (1973), 305-308.

[10] T. R. Rockafellar, Convex analysis (Princeton: Princeton University Press, 1970).

[11] S. Schaible, "Parameter free convex equivalent and dual programs of fractional programming problems", Zeitschrift für Operations Research 18 (1974), 187-196.

[12] S. Schaible, "Fractional programming. I. Duality", Management Science 22 (1976), 858-862.

[13] S. Schaible, "Duality in fractional programming: a unified approach", Operations Research 24 (1976), 452-561.

School of Mechanical and Industrial Engineering

University of N.S.W.

Kensington, N.S.W. 2033

Australia 